\title{
REPERCUSIONES EMOCIONALES DE LA VIOLENCIA ESCOLAR: INFLUENCIA EN LA INTELIGENCIA EMOCIONAL
}

\section{EMOTIONAL REPERCUSSIONS OF SCHOOL VIOLENCE: INFLUENCE ON EMOTIONAL INTELLIGENCE}

\author{
NIEVES GUTIÉRREZ ÁNGEL ${ }^{1}$
}

Cómo referenciar este artículo/How to reference this article:

Gutiérrez Ángel, N. (2019). Repercusiones Emocionales de la Violencia Escolar: Influencia en la Inteligencia Emocional [Emotional Repercussions of School Violence: Influence on Emotional intelligence]. Acción Psicológica, 16(1), 143-156. https://doi.org/10.5944/ap.16.1.22555

\section{Resumen}

Entender la violencia escolar o bullying como un hecho que no es fijo y estable, sino como un proceso mediante el cual una persona se ve agredida física o psicológicamente por otra más fuerte, dando lugar a un situación de desventaja y dañina, es la conceptualización que mayor aceptación posee en la actualidad. Junto con ella, se distinguen tres grandes protagonistas, como son el agresor, la víctima y observador. El objetivo de este trabajo es identificar la influencia que ha tenido el estar implicado en la dinámica del bullying en los niveles de Inteligencia Emocional de cada uno de los tres roles existentes. El método empleado se corresponde con un diseño ex post facto, de carácter retrospectivo y comparativo. Y la muestra está compuesta por estudiantes de tercer y cuarto curso de sus estudios de Grado, de la Universidad de Almería (España). Los resultados aportados por este trabajo ponen de manifiesto que tanto las no víctimas como los no agresores poseen niveles más altos en cada una de las dimensiones que componen la Inteligencia Emocional. Concluyendo en que, por tanto, el verse implicado en algún episodio relacionado con la violencia escolar, ya sea como víctima o como agresor influye de manera negativa en los niveles de Inteligencia Emocional, ya que disminuye los mismos. En el caso de los observadores, dependiendo del posicionamiento que adopten ante estos hechos poseen unos niveles más altos o más bajos de Inteligencia Emocional.

Palabras clave: Consecuencias; Inteligencia emocional; Roles; Violencia escolar.

Correspondence address [Dirección para correspondencia]: Nieves Gutiérrez Ángel. Universidad de Almería, España. Email:nga212@ual.es

ORCID Nieves Gutiérrez Ángel (http://orcid.org/0000-0003-4705-3225).

${ }^{1}$ Universidad de Almería, España.

Recibido: 6 de septiembre de 2018.

Aceptado: 17 de mayo de 2019. 


\begin{abstract}
Understanding school violence or bullying as a fact that is not fixed and stable, but as a process by which a person is physically or psychologically attacked by a stronger one, leading to a disadvantageous and harmful situation, is the conceptualization that greater acceptance has nowadays. Along with it, there are three main protagonists, such as the aggressor, the victim and the observer. The objective of this work is to identify the influence that has been involved in the dynamics of bullying in the levels of Emotional Intelligence of each of the three existing roles. The method used corresponds to an ex post facto design, of a retrospective and comparative nature. And the sample is composed of third and fourth year students of their degree studies, from the University of Almeria (Spain). The results provided by this work show that both nonvictims and non-aggressors have higher levels in each of the dimensions that make up the Emotional Intelligence. Concluding that, therefore, being involved in an episode related to school violence, either as a victim or as an aggressor, negatively influences Emotional Intelligence levels, since it decreases them. In the case of observers, depending on the positioning they adopt in the face of these events, they have higher or lower levels of Emotional Intelligence.
\end{abstract}

Keywords: Bullying; Emotional intelligence; Consequences; Roles.

\section{Introducción}

La dinámica del bullying, no es una dinámica fija y estable, sino que es un proceso de acoso que se desarrolla mediante cinco fases que hacen alusión al proceso en el que se ve implicada una víctima ante una situación de acoso escolar (Piñuel y Oñate, 2007). Dicho proceso, comienza a partir de la incidencia, a través del propio acoso y estigmatización. Una vez ocurrido, ocurren tanto el origen como la latencia del daño provocado a la víctima, y como consecuencia de ello, las manifestaciones somáticas o psicológicas que la víctima experimenta, traduciéndose en sentimientos de inferioridad, o exclusión.

Además, también son destacables los efectos de estas conductas violentas en cuanto a su difusión en el contexto social. Siguiendo a Sullivan, Cleary y Sullivan (2005) también establecen cinco niveles en cuanto al proceso de propagación que conlleva una agresión. En este sentido, comparte con el proceso anterior el primer nivel o paso, que se trata de la incidencia del acoso propiamente dicho. A continuación, intervienen en el mismo todo el personal que se encuentra alrededor de la víctima, tanto en el contexto escolar (compañeros, profesores...) como en el contexto familiar. Por lo que, a partir de este momento, deja de ser un acto entre el agresor y la víctima solamente. Los siguientes niveles están relacionados con la toma en consideración de los hechos, y en la propuesta de distintos tipos de intervenciones ante la ocurrencia de los mismos.

Es por tanto Olweus (1978), quien define e identifica tanto al bullying como a sus protagonistas por primera vez (Ruiz, Riuró y Tesouro, 2015), es decir, agresor, víctima y observador. Atendiendo a cada uno de ellos, el agresor es quien realiza la acción dañina, la víctima quien es sometido a tal acción y el perjudicado, y el observador, que es un mero curioso cómplice de la situación.

En una aportación más reciente, Avilés (2006) establece la importancia de tales roles en la dinámica y desarrollo de estos episodios, la cual está caracterizada por relaciones asimétricas entre los agresores, que intentan dominar e intimidar a la víctima y convertir al espectador en un cómplice activo o silencioso que apoya esta situación (Quintana, Montgomery y Malaver, 2009). Siendo por lo tanto los tres roles protagonistas que forman parte de la violencia escolar como un proceso (Polo del Río, Mendo, Fajardo y León del Barco, 2017).

Al respecto, destacan las aportaciones de otros autores, como Cerezo (2002), quien posee una visión social de este fenómeno y junto con ello, distingue tres tipos de subgrupos sociométricos que se pueden encontrar dentro de un mismo grupo: El primero de ellos, formado por alumnos que gozan de popularidad entre el resto. El segundo grupo compuesto por alumnos aislados, que suelen quedar al 
margen. Y, el tercer grupo, integrado por aquellos alumnos rechazados que no gozan de popularidad.

Si nos centramos en los episodios violentos que suceden en el contexto escolar, parece claro identificar cada uno de estos grupos con los roles a los que venimos refiriéndonos. $\mathrm{Y}$ es que, desde esta visión, se considera que la posición, la ascendencia y el nivel de social tienen una gran relevancia en el sostenimiento y fomento del bullying, relacionándose cada uno de estos tres subgrupos con un rol: Agresor, víctima y observador (Salmivalli, 2010).

A pesar de que la delimitación de los tres roles formulados por Olweus (1991) gozan de una amplia aceptación, hemos de mencionar que existen otras clasificaciones de los roles implicados en la violencia escolar, como es el caso de las aportaciones de Stephenson y Smith (1987) quienes distinguen cinco roles: Agresores, agresores ansiosos, víctimas, víctimas provocativas y agresores-víctimas. O la aportación de Salmivalli, Lagerspetz, Björkqvist, Österman y Kaukiainen (1996), diferenciando seis roles distintos: Agresor, reforzador del agresor, ayudante del agresor, defensor de la víctima, ajeno y víctima. Así como también, es destacable la clasificación de Ortega y Mora-Merchán (2000), quienes distinguen entre agresores, víctimas, agresores victimizados y espectadores.

Por lo tanto, indistintamente de las aportaciones a las que hagamos alusión, es clara la identificación de tres grandes roles implicados en la violencia escolar. Entrando de lleno en las características de cada uno de ellos.

En cuanto a los agresores, son considerados alumnos impulsivos, con escaso autocontrol (Martos y Del Rey, 2013), una baja tolerancia a la frustración, y dificultades para cumplir las normas (Díaz-Aguado, 2006), una baja capacidad de autocrítica, escaso ajuste social, amplia necesidad de autoafirmación (Albadalejo, 2011). Los cuales gozan agrediendo a otra persona, sin motivo alguno, asociando la agresión a la popularidad, o en la creencia de que algunos compañeros son merecedores de la agresión, o por simple diversión.

En lo que respecta a las características físicas, generalmente son hombres de mayor edad que sus víctimas, y fí- sicamente fuertes (Ruiz et al., 2015), con una actitud negativa ante la escuela (Martos y del Rey, 2013), así como un bajo rendimiento académico y relaciones negativas con el profesorado (Díaz-Aguado, 2006).

Por otro lado, las víctimas suelen ser alumnos aislados, sin apoyo, con bajo nivel de aceptación y alto nivel de rechazo (Sierra, 2009), ansiosos, vulnerables, indefensos e inseguros, no poseen la capacidad de reacción ante la situación en muchas ocasiones, y poseen una baja autoestima (González, 2012; Rose, Monda Amaya y Espelage, 2011). Esta falta de inseguridad y asertividad, les conduce por lo tanto a tener una imagen muy negativa sobre ellos mismos (Albadalejo, 2011), un tipo de personalidad pobre, o algún rasgo físico distinto (Armero, Bernardino, y Bonet, 2011; Díaz-Aguado, Martínez y J. Martín, 2013).

En lo que respecta al terreno escolar, el verse implicado como víctima puede influir o no en el rendimiento académico, y un sentimiento mayor de miedo en el centro escolar acompañado de indefensión (Martos y del Rey, 2013). Autores como Rigby (1996) van más allá, argumentando incluso que las víctimas pueden llegar a padecer trastornos psicosomáticos, como dolores, así como no querer asistir al centro escolar, unos bajos rendimientos académico, dormir mal, retraerse... acompañados en la mayoría de las ocasiones del silencio imperante ante lo que está sucediendo.

En el caso de los observadores, pueden posicionarse de dos grandes maneras: Ser meros espectadores de la situación que contemplan sin hacer nada ante ello (Ruiz et al., 2015), apoyando con su silencio, de manera encubierta y pasiva al agresor (Cerezo y Sánchez, 2013) y no enfrentándose al mismo, bien por miedo (Trautmann, 2008) o bien porque consideran que la situación de conflicto no va con ellos (Molina y Vecina, 2015), dotando a los agresores de cierto apoyo implícito (Díaz-Aguado, Martínez y G. Martín, 2004). O por el contrario, poseer un papel mucho más activo en el que muestren su desagrado ante los hechos y apoyen a la víctima (Cuevas y Marmolejo, 2016). Siendo en este caso la adopción de esta postura esencial a la hora de detener el desarrollo del bullying (Garaigordobil y Aliri, 2013; Polo del Río et al., 2017). 
Además de las características propias de cada rol, si revisamos la literatura existente, también aparecen en la misma las consecuencias a las que están expuestos cada uno de estos roles (Garaigordobil, 2017; Lara-Ros, Rodríguez-Jiménez, Martínez-González y Piqueras, 2017; Larrañaga, Yubero, Ovejero y Navarro, 2013; Morales, Yubero y Larrañaga, 2016). Las cuales se corresponden con la tendencia a que, a través del empleo de la violencia se llegue a la delincuencia en el caso de los agresores (Ortega, 2000). La exclusión y las dificultades para relacionarse por parte de las victimas (Martínez-Otero, 2005). Así como el fomento de sentimientos de desvaloración, ansiedad, insatisfacción, culpa, soledad o fobias e inseguridad que dan lugar a la aparición y afianzamiento de sentimientos negativos sobre ellas mismas (Morris, Zhang y Bondy, 2006; Storch et al., 2005) o comportamientos negativos asociados a la hostilidad y la agresividad (Garaigordobil, 2015; Santos y Romera, 2013) y la presencia de niveles bajos de empatía (Cerezo y Sánchez, 2013) que pueden dar lugar a alternaciones psicosociales y psicopatológicas (Garaigordobil, 2011) fundamentados a partir de una idea errónea sobre la violencia escolar como algo normal (Puértolas y Montiel, 2017) en el caso de los agresores.

En cualquier caso, las consecuencias de estos actos afectan a los tres roles implicados en esta dinámica a la que venimos haciendo alusión (Inglés et al., 2014), destacando entre ellas la gran probabilidad de sufrir desajustes psicosociales y/o trastornos psicopatológicos (Ruiz et al., 2015). Pese a que la peor de las consecuencias de este fenómeno es el suicidio, no menos importantes son el resto de consecuencias a las que puede dar lugar, ya que, aunque no son tan desmedidas, sí interceden en la salud, la calidad de vida, el bienestar y el desarrollo personal de los implicados (Garaigordobil, 2013).

Por todo ello, el objetivo de este trabajo es identificar la influencia que ha tenido el estar implicado en la dinámica del bullying en los niveles de Inteligencia Emocional de cada uno de los tres roles existentes.

\section{Método}

El método empleado es correlacional correspondiente con un diseño ex post facto, de carácter retrospectivo y comparativo, ya que se comparan los tres roles implicados en la dinámica del bullying (agresor, víctima y observador) con una variable dependiente, que en este caso, se corresponden con cada uno de los niveles de la Inteligencia Emocional.

\section{Participantes}

La muestra está compuesta por estudiantes de tercer y cuarto curso de sus estudios de Grado, en concreto, se corresponden con el Grado de Educación Social de la Universidad de Almería (España), los cuales han sido seleccionados mediante un muestreo aleatorio simple, siendo un total de 175 sujetos.

La edad media de la muestra es de 25.79 años, con una desviación típica $D T=8.01$. En cuanto al sexo, el $22.9 \%$ $(N=40)$ son hombres, y el $77.1 \%(N=135)$ mujeres. Y en cuanto al curso, pertenecen al tercer curso el $42.9 \%$ $(N=75)$ y al cuarto curso el $57.1 \%(N=100)$.

\section{Instrumentos}

Se han empleado tres instrumentos en este estudio. El primero de ellos, un instrumento elaborado "ad hoc" cuyo objetivo fue recabar información de tipo sociodemográfico sobre la muestra, en cuanto a cuestiones como la edad, el sexo, la titulación, o la formación en el contexto educativo.

El segundo instrumento empleado es el Trait Meta Mood Scale-24 (TMMS-24) elaborado por Fernández-Berrocal, Extremera, y Ramos (2004) el cual se corresponde con una adaptación del Trait Meta-MoodScale (TMMS) elaborado por Salovey, Mayer, Goldman, Turvey y Palfai (1995) cuya finalidad es evaluar la Inteligencia Emocional percibida por el sujeto. El cual está compuesto por veinticuatro ítems que evalúan las tres dimensiones de la Inteligencia Emocional aportadas por el modelo de Salovey y Mayer (1990): percepción, comprensión y regulación de las emociones. Siendo ocho los ítems que evalúan cada 
uno de esas dimensiones (Rey y Extremera, 2012; Rueda y López, 2013).

Las propiedades psicométricas de este instrumento son apropiadas (Fernández-Berrocal et al., 2004). En concreto, el $\alpha$ de Cronbach es de $=.90$ para la percepción de las emociones, $=.90$ para la comprensión de las emociones, y $=.86$ para la regulación de las emociones (Cazalla-Luna, Ortega-Álvarez y Molero, 2015), al igual que posee una fiabilidad test-retest favorable, atención $=.60$; clari$\mathrm{dad}=.70$ y reparación $=.83$ (Extremera y Fernández-Berrocal, 2005). En el caso de este trabajo Alpha de Cronbach $=.94$ para la atención emocional, Alpha de Cronbach $=.98$ para la comprensión de las emociones, $\mathrm{y}$ Alpha de Cronbach $=.89$ para la regulación de las emociones.

El tercer instrumento empleado es el Cuestionario sobre maltrato entre iguales en la escuela (Nicolaides, Toda, y Smith, 2002). Cuyo objetivo principal es evaluar distintos aspectos de la violencia escolar, tomando en este caso como referencia la visión de los profesionales de la educación, por medio de siete bloques que hacen alusión tanto a la experiencia personal, los conocimientos y creencias sobre el bullying, la percepción e identificación de características propias tanto de los agresores, como de las víctimas, la intervención ante estos hechos, las estrategias empleadas y recomendadas al alumnado ante ello, y la valoración de la propia formación como profesionales de cara a esta temática (Benítez, Berbén y Fernández, 2006). Dando lugar todo ello, a un total de cuarenta y cinco ítems, organizados mediante quince cuestiones. En concreto, para este trabajo, de todos los ítems que componen el mismo, se han utilizado únicamente los que están destinados a la recogida de información que esté relacionada con la propia experiencia como agresor, víctima y observador.

El Alpha de Cronbach de este instrumento obtenido en otros trabajos $=0.94$ (Benítez et al., 2006). Mientras que en el caso de este trabajo el Alpha de Cronbach = .97.

\section{Procedimiento}

Se facilitó al alumnado mediante el aula virtual de distintas asignaturas un enlace en el que podían acceder a la información y realización del cuestionario. Previamente a ello, dicho cuestionario había sido creado en la plataforma de encuestas "Lime Survey".

\section{Análisis de datos}

El análisis de datos se ha realizado mediante el análisis estadístico descriptivo en cuanto a cada rol. Además de las pruebas $t$ de Student para grupos independientes, en este caso aplicada con variables como cada uno de los citados roles. La realización de todas estas pruebas tuvo como finalidad no solamente medir las puntuaciones obtenidas en cuanto a la Inteligencia Emocional percibida, sino también analizar la posible existencia de diferencias entre cada grupo. Para finalizar, en aquellas situaciones en las que se encontraron diferencias estadísticamente significativas, se calculó la $d$ de Cohen (1988) para medir el tamaño del efecto. Todo ello a través de la utilización del programa de análisis estadístico SPSS en su versión 23.

\section{Resultados}

\section{Frecuencias de cada uno de los roles im- plicados en la dinámica del bullying}

En primer lugar, se pidió a la muestra que señalara entre nunca, ocasionalmente, algunas veces o frecuentemente las veces que se habían visto implicados en algún episodio relacionado con la violencia escolar, ya fuera como víctimas, como agresores, o como observadores.

En el caso del rol de víctima, el $26.9 \%(N=47)$ de la muestra manifestó no haber sido nunca víctima. El $26.9 \%$ $(N=47)$ manifestó haber sido víctima en algunas ocasiones (dos o tres veces). El $7 \%(N=4)$ respondió haberlo sido pocas veces cada año o a menudo durante un mismo año. Y $42.3 \%(N=74)$ respondieron haber sido víctimas de forma frecuente.

En el caso del rol, de agresor, el $67.4 \%(N=118)$ nunca había sido agresor, mientras que un $12.6 \%(N=22)$ lo habían sido ocasionalmente, un $20 \%(N=35)$ lo había 
Tabla 1

Roles implicados en la violencia escolar

\begin{tabular}{|c|c|c|c|c|c|c|}
\hline \multirow[b]{2}{*}{ Frecuencia } & \multicolumn{2}{|c|}{ Víctimas } & \multicolumn{2}{|c|}{ Agresores } & \multicolumn{2}{|c|}{ Observadores } \\
\hline & $N$ & $\%$ & $\vec{N}$ & $\%$ & $\bar{N}$ & $\%$ \\
\hline Nunca & 47 & $26.9 \%$ & 118 & $67.4 \%$ & 27 & $15.4 \%$ \\
\hline Ocasionalmente & 47 & $26.9 \%$ & 22 & $12.6 \%$ & 144 & $82.3 \%$ \\
\hline Algunas veces & 7 & $4 \%$ & 35 & $20 \%$ & 2 & $1.1 \%$ \\
\hline Frecuentemente & 74 & $42.3 \%$ & - & - & 2 & $1.1 \%$ \\
\hline
\end{tabular}

sido algunas veces, y un $0 \%(N=0)$ lo habían sido frecuentemente.

Mientras que, en el caso de los observadores, el $15.4 \%$ $(N=27)$ no lo había sido nunca, el $82.3 \%$. $(N=144)$ de forma ocasional, el $1.1 \%(N=2)$ lo habían sido algunas veces, y el $1.1 \%(N=2)$ frecuentemente. En la tabla $1 \mathrm{se}$ presentan las frecuencias de respuesta de cada uno de los tres roles.

Una vez analizadas las frecuencias de cada uno de los roles implicados en la dinámica del bullying, los mismos fueron recodificados en nuevas variables de cara a identificar si cada uno de los roles habían sido desempeñados o no. De tal manera, nunca fue considerado como no, y ocasionalmente, algunas veces y frecuentemente como sí. De tal manera, del total de la muestra, un $26.9 \%(N=47)$ no habían sido víctimas, y un $73.1 \%(N=128)$ sí lo habían sido. Un $67.4 \%(N=118)$ no habían sido agresores, y un $32.6 \%(N=57)$ sí lo habían sido. Y un $1.1 \%(N=27)$ no habían sido observadores, y un $84.6 \%(N=148)$ sí lo habían sido.

Tal y como se observa en la Tabla 2 , el rol más ejercido por la muestra es el de observador, y en segundo lugar el de víctima. Mientras que el menos ejercido se corresponde con el rol de agresor.

Tabla 2

Roles implicados en la violencia escolar (recodificados

\begin{tabular}{lcccccc}
\hline & \multicolumn{2}{c}{ Víctimas } & \multicolumn{2}{c}{ Agresores } & \multicolumn{2}{c}{ Observadores } \\
\hline Frecuencia & \multicolumn{1}{c}{$\boldsymbol{N}$} & $\%$ & \multicolumn{1}{c}{$\boldsymbol{N}$} & $\%$ & \multicolumn{1}{c}{} & $\%$ \\
\hline No & 47 & 26.9 & 118 & 67.4 & 27 & 15.4 \\
Sí & 128 & 73.1 & 57 & 32.6 & 148 & 84.6 \\
\hline
\end{tabular}

\section{Influencia del desempeño de cada rol en los niveles de Inteligencia Emocional}

De cara a conocer la posible influencia del rol desempeñado en los niveles de Inteligencia Emocional se realizaron con cada uno de ellos una prueba $t$. De tal manera, en el caso de las víctimas y las no víctimas (Tabla 3) los resultados indican que no existen diferencias significativas entre ambos en atención emocional $\left(t_{(175}=1.92\right.$, $p=.05)$, claridad emocional $\left(t_{(175)}=1.59, p=.11\right)$, ni en reparación emocional $\left(t_{(175}=0.81, p=.41\right)$. Es decir, los resultados obtenidos a partir de la prueba $t$ para muestras independiente, no muestran diferencias estadísticamente

Tabla 3

Inteligencia Emocional. Descriptivos y prueba t según rol (no víctima/ víctima)

\begin{tabular}{|c|c|c|c|c|c|c|c|c|}
\hline \multirow{3}{*}{ TMMS-24 } & \multicolumn{6}{|c|}{ Inteligencia emocional } & \multirow{3}{*}{$t$} & \multirow{3}{*}{$p$} \\
\hline & \multicolumn{3}{|c|}{ No víctima } & \multicolumn{3}{|c|}{ Víctima } & & \\
\hline & $\mathbf{N}$ & $M$ & $D T$ & $\mathbf{N}$ & $M$ & $D T$ & & \\
\hline Atención emocional & 42 & 31.69 & 4.40 & 123 & 29.05 & 8.48 & 1.92 & .05 \\
\hline Claridad emocional & 42 & 31.45 & 4.53 & 123 & 29.15 & 8.95 & 1.59 & .11 \\
\hline Reparación emocional & 42 & 29.21 & 5.13 & 123 & 28.17 & 7069 & .81 & .41 \\
\hline
\end{tabular}


Tabla 4

Inteligencia Emocional. Descriptivos y prueba t según rol (no agresor/ agresor)

\begin{tabular}{|c|c|c|c|c|c|c|c|c|c|}
\hline \multirow{3}{*}{ TMMS-24 } & \multicolumn{6}{|c|}{ Inteligencia emocional } & \multirow{3}{*}{$t$} & \multirow{3}{*}{$p$} & \multirow{3}{*}{$d$} \\
\hline & \multicolumn{3}{|c|}{ No agresor } & \multicolumn{3}{|c|}{ Agresor } & & & \\
\hline & $\boldsymbol{N}$ & $M$ & $D T$ & $\boldsymbol{N}$ & $M$ & $D T$ & & & \\
\hline Atención emocional & 112 & 30.93 & 6.73 & 53 & 27.16 & 9.03 & $2.99^{*}$ & .00 & .47 \\
\hline Claridad emocional & 112 & 30.29 & 7.79 & 53 & 28.56 & 8.68 & 1.28 & .20 & - \\
\hline Reparación emocional & 112 & 28.55 & 6.80 & 53 & 28.20 & 7.82 & .29 & .77 & - \\
\hline
\end{tabular}

significativas en ninguna dimensión entre aquellos participantes que han sido víctimas y los que no lo han sido.

Por otro lado, en cuanto a los que habían sido o no agresores, los resultados muestran que no existen diferencias en claridad emocional $\left(t_{(175}=1.28, p=.20\right)$, ni en reparación emocional $\left(t_{(175}=0.29, p=.77\right)$. Pero sin embargo, los resultados obtenidos aportan la existencia de diferencias estadísticamente significativas en atención emocional $\left(t_{(175)}=2.99, p<.001, d=.47\right)$. Y, por tanto, se puede afirmar que los que no han sido agresores $(M=30.93)$ obtienen puntuaciones más elevadas que los que sí lo han sido $(M=27.16)$. Siendo El tamaño del efecto moderado $(d<.50)$, Tal y como muestra la Tabla 4.

En tercer lugar, con respecto a los observadores (tabla 5), los resultados ponen de manifiesto el hecho de que no existen diferencias estadísticamente significativas en claridad emocional $\left(t_{(175)}=-.67, p=.5\right)$, ni en reparación emocional $\left(t_{(175)}=1.69, p=.05\right)$. Sin embargo, si existen diferencias significativas cuando se trata de la claridad emocional $\left(t_{(175)}=2.72, p<.001, d=.7\right)$. Y, por tanto, se puede afirmar que los que no han sido observadores $(M=33.55)$ obtienen puntuaciones más elevadas que los

Tabla 5

Inteligencia emocional. Descriptivos y prueba t según rol (no observador/observador)

\begin{tabular}{|c|c|c|c|c|c|c|c|c|c|}
\hline \multirow{3}{*}{ TMMS-24 } & \multicolumn{6}{|c|}{ Inteligencia emocional } & \multirow{3}{*}{$t$} & \multirow{3}{*}{$p$} & \multirow{3}{*}{$d$} \\
\hline & \multicolumn{3}{|c|}{ No observador } & \multicolumn{3}{|c|}{ Observador } & & & \\
\hline & $N$ & $M$ & $D T$ & $N$ & $M$ & $D T$ & & & \\
\hline Atención emocional & 27 & 28.81 & 6.88 & 138 & 29.90 & 7.89 & -.67 & .5 & - \\
\hline Claridad emocional & 27 & 33.55 & 6.37 & 138 & 28.99 & 8.22 & $2.72^{* *}$ & .00 & .62 \\
\hline Reparación emocional & 27 & 30.88 & 6.23 & 138 & 27.96 & 7.21 & .69 & .05 & - \\
\hline
\end{tabular}

que sí lo han sido $(M=28.99)$. Siendo el tamaño del efecto moderado $(d<.50)$.

\section{Discusión y conclusiones}

Los resultados obtenidos en este trabajo ponen de manifiesto el hecho de que no existen diferencias entre la Inteligencia Emocional y el haber sido víctima o no haberlo sido. No obstante, hemos de ser cautelosos con estos resultados, ya que en todas las dimensiones de la Inteligencia Emocional las no víctimas obtienen mayores niveles tanto en atención, como en claridad y regulación emocional, resultados similares a los aportados por otros trabajos que van en esta misma dirección (Boulton, 1997; Craig, Pepler y Atlas, 2000; Dake, Price, Telljohann y Funk, 2003; Mishna, Sacarcello, Pepler y Weiner, 2005; Raj, Aluede, McEachern y Kenny, 2005).

Por otro lado, en cuanto al rol de agresor, son los no agresores los que mayores niveles presentan en atención, claridad y regulación emocional, existiendo incluso diferencias estadísticamente significativas en el caso de la atención emocional por parte de los mismos. Ante lo que queda demostrado que los agresores se corresponden con 
personas con una baja capacidad de autocrítica, escaso ajuste social, amplia necesidad de autoafirmación (Albadalejo, 2011).

Por último, en cuanto al rol de observador, obtienen mayores niveles de reparación y claridad emocional (esta última significativa) que los observadores. Y solamente se da el caso contrario, en el que los observadores presentan mayores niveles, en el caso de la atención emocional. Este resultado, en gran parte puede ser explicado en cuanto al posicionamiento que desde este rol se puede adoptar, teniendo un papel mucho más activo en el que muestran su desagrado ante los hechos y muestran apoyo a la víctima (Cuevas y Marmolejo, 2016). Siendo en este caso la adopción de esta postura esencial a la hora de detener el desarrollo del bullying (Garaigordobil y Aliri, 2013; Polo del Río et al., 2017).

En conclusión, los resultados aportados por este trabajo ponen de manifiesto que tanto las no víctimas como los no agresores poseen niveles más altos en cada una de las dimensiones que componen la Inteligencia Emocional. Hipótesis que si abordamos con detenimiento nos conduce a pensar que el verse implicado en algún episodio relacionado con la violencia escolar, ya sea como víctima o como agresor influye de manera negativa en los niveles de Inteligencia Emocional, ya que disminuye los mismos.

Sin dejar de lado a los observadores, quienes dependiendo del posicionamiento que adopten ante estos hechos poseen unos niveles más altos o más bajos de dichos niveles. Recordemos que son diversos los estudios que ponen de manifiesto el hecho de que la adopción de un papel de mero espectador sin hacer nada ante ello (Ruiz et al., 2015), apoya con su silencio, de manera encubierta y pasiva al agresor (Cerezo y Sánchez, 2013) dotando al mismo de cierto apoyo implícito (Díaz-Aguado et al., 2004). Y cuyas consecuencias se encuentran estrechamente relacionadas con los comportamientos negativos asociados a la hostilidad y la agresividad (Garaigordobil, 2015; Santos y Romera, 2013) y la presencia de niveles bajos de empatía (Cerezo y Sánchez, 2013).

\section{Referencias}

Albadalejo, A. (2011). Evaluación de la violencia escolar en educación infantil y primaria [Evaluation of School Violence in Infant and Primary Education]. Alicante, España: Universidad de Alicante.

Armero, P., Bernardino, B. y Bonet, C. (2011). Acoso escolar [Bullying]. Revista Pediatría de Atención Primaria, 13(52), 661-670. https://doi.org/10.4321/S1139-7632201100060001 6

Avilés, J. M. (2006). Bullying: el maltrato entre iguales. Agresores, víctimas y testigos en la escuela [Bullying: Aggressors, Victims and Eyewitnesses in School]. Salamanca, España: Amarú.

Benítez, J. L., Berbén, A. G. y Fernández, M. (2006). El maltrato entre alumnos: Conocimientos, percepciones y actitudes de los futuros docentes. [Student Abuse: Knowledge, Perceptions and Attitudes of Future Teachers]. Revista de Investigación Educativa, 24(2), 329-352.

Boulton, M. J. (1997). Teachers' Views on Bullying Definitions, Attitudes, and Ability to Cope. British Journal of Educational Psychology, 67(2), 223233. https://doi.org/10.1111/j.2044-8279.1997.tb0 1239.x

Cazalla-Luna, N., Ortega-Álvarez, F. y Molero, D. (2015). Autoconcepto e inteligencia emocional de docentes en prácticas [Self-concept and Emotional Intelligence of Teachers in Practice]. Revista Electrónica de Investigación y Docencia, 14, 151-164.

Cerezo, F. (2002). Bull-S: Test de evaluación de la agresividad entre escolares. Manual de referencia [Bull-S: Test to Evaluate Aggressiveness Among Schoolchildren. Reference Manual]. Vizcaya, España: ALBOR-COHS.

Cerezo, F. y Sánchez, C. (2013). Eficacia del programa CIP para la mejora de la convivencia escolar y la prevención del bullying en alumnos de Educación 
Primaria [Effectiveness of the IPC Programme in Improving the School Coexistence and Bullying Prevention in Students of Primary Education. Apuntes de Psicología, 31(2), 173-181.

Cohen, J. (1988). Statistical Power Analysis for the Behavioral Sciences. Hillsdale, NJ: Erlbaum.

Craig, W. M., Pepler, D. J. y Atlas, R. (2000). Observations of Bullying in the Playground and in the Classroom. School Psychology International, 21(1), 22-36. https://doi.org/10.1177/014303430 0211002

Cuevas, M. C. y Marmolejo, A. (2016). Observadores: un rol determinante en el acoso escolar [Bystanders: A Crucial Role in Bullying]. Pensamiento Psicológico, 14(1), 89-102. https://doi.org/10. 11144/Javerianacali.PPSI14-1.orda

Dake, J. A., Price, J. H., Telljohann, S. K. y Funk, J.B. (2003). Teacher Perceptions and Practices Regarding School Bullying Prevention. Journal of School Health, 73(9), 347-355. https://doi.org/10. 1111/j.1746-1561.2003.tb04191.x

Díaz-Aguado, M. J. (2006). Sexismo, violencia de género y acoso escolar. Propuestas para una prevención integral de la violencia [Sexism, Gender-Based Violence and Bullying. Proposals for Comprehensive Violence Prevention]. Revista de Estudios de Juventud, 73, 38-57.

Díaz-Aguado, M. J., Martínez, R. y Martín, G. (2004). Prevención de la violencia y la exclusión socia [Violence Prevention and Social Exclusión]. Madrid, España: Instituto de la Juventud.

Díaz-Aguado, M. J., Martínez, R. y Martín, J. (2013). El acoso entre adolescentes en España. Prevalencia, papeles adoptados por todo el grupo y características a las que atribuyen la victimización [Bullying among Adolescents in Spain, Prevalence, Participant Roles and Characteristics Attributable to Victimization by Victims and Aggressors. Revista de
Educación, 362, 348-379. https://doi.org/104438/1988-592X-RE-2011-362-164

Extremera, N. y Fernández-Berrocal, P. (2005). Inteligencia emocional percibida y diferencias individuales en el meta-conocimiento de los estados emocionales: una revisión de los estudios con el TMMS [Perceived Emotional Intelligence and Individual Differences in the Meta-knowledge of Emotional States: A Review of the Studies with the Trait Meta-Mood Scale]. Ansiedad y Estrés, 11(2-3), 101-122.

Fernández-Berrocal, P., Extremera, N. y Ramos, N. (2004). Validity and Reliability of the Spanish Modified Version of the Trait Meta-Mood Scale. Psychological Reports, 94(3), 751-755. https://doi.org/10.2466/PR0.94.3.751-755

Garaigordobil, M. (2011). Prevalencia y consecuencias del cyberbullying [Prevalence and Consequences of Cyberbullying: A Review]. International Journal of Psychology and Psychological Therapy, 11(2), 233-254.

Garaigordobil, M. (2013). Screening de acoso entre iguales [Screening of Harassment among Equals]. Madrid, España: TEA.

Garaigordobil, M. (2015). Ciberbullying en adolescentes y jóvenes del País Vasco: cambios con la edad [Cyberbullying in Adolescents and Youth in the Basque Country: Changes with Age]. Anales de Psicología, 31(3), 1069-1076. http://dx.doi.org/ 10.6018/analesps.31.3.179151

Garaigordobil, M. (2017). Conducta antisocial: conexión con bullying/cyberbullying y estrategias de resolución de conflictos [Antisocial behavior: Connection with bullying/cyberbullying and conflict resolution]. Psychosocial Intervention, 26, 47-54. https://doi.org/10.1016/j.psi.2015.12.002

Garaigordobil, M. y Aliri, J. (2013). Ciberacoso ("cyberbullying") en el país vasco: Diferencias de sexo en víctimas, agresores y observadores [Cyberbullying 
in the Basque Country: Sex Differences in Victims, Aggressors and Observers]. Psicología Conductual, 21(3), 461-474.

González, V. (2012). Bullying y acoso escolar durante el desarrollo de las clases de educación física, revisión teórico-conceptual [Bullying and School Harrasment during the Development of Physical Education Classes, Theoretical-Conceptual Review]. Emásf. Revista Digital de Educación Física, 6, 16.

Inglés, C., Torregrosa, M. S., García-Fernández, J. M., Martínez-Monteagudo, M. C., Esteve, E. y Delgado, B. (2014). Conducta agresiva e inteligencia emocional en la adolescencia [Aggressive Behavior and Emotional Intelligence in Adolescence]. European Journal of Education and Psychology, 7(1), 29-41. https://doi.org/10.30552/ejep.v7i1.97

Lara-Ros, M. R., Rodríguez-Jiménez, T., Martínez-González, A. E. y Piqueras, J. A. (2017). Relación entre el bullying y el estado emocional y social en niños de educación primaria [Relationship between Bullying and Emotional and Social Status among Primary Scholars]. Revista de Psicología Clínica con Niños y Adolescentes, 4(1), 59-64.

Larrañaga, E., Yubero, S., Ovejero, A. y Navarro, R. (2013). Validación de la versión española de la Gender Role Conflict Scale for Adolescents (GRCS-A) [Validation of the Spanish Version Gender Role Conflict Scale for Adolescents (GRCSA)]. Anales de Psicología, 29(2), 549-557. https://doi.org/10.6018/analesps.29.2.143341

Martínez-Otero, V. (2005). Conflictividad escolar y fomento de la convivencia [School Conflict and Promotion of Coexistence]. Revista Iberoamericana de Educación, 38, 33-52. https://doi.org/10.35362/rie 380829

Martos, A. y Del Rey, R. (2013). Implicación del alumnado con necesidades específicas de apoyo educativo en bullying [Involvement of Students with
Specific Needs for Educational Support in Bullying]. Apuntes de Psicología, 31(2), 183-190.

Mishna, F., Sacarcello, I., Pepler, D., y Weiner, J. (2005). Teachers' Understanding of Bullying. $\mathrm{Ca}$ nadian Journal of Education, 28(4), 718-738. https://doi.org/10.2307/4126452

Molina, J. A., y Vecina, P. (2015). Bullying, ciberbullying y sexting. ¿Cómo actuar ante una situación de acoso? [Bullying, Cyberbullying, and Sexting. How to Deal with Harassment?] Madrid, España: Pirámide.

Morales, J. F., Yubero, S. y Larrañaga, E. (2016). Gender and Bullying: Application of a Three-factor Model of Gender Stereotyping. Sex Roles, 74(3-4), 169-180. https://doi.org/10.1007/s11199-015-046 3-3

Morris, E. B., Zhang, B., y Bondy, S. J. (2006). Bullying and Smoking: Examining the Relationships in Ontario Adolescents. Journal of Scholl Health, 76(9), 465-470. https://doi.org/10.1111/j.1746-1561.200 6.00143.x

Nicolaides, S., Toda, Y. y Smith, P.K. (2002). Knowledge and Attitudes about School Bullying in Trainee Teachers. British Journal of Educational Psychology, 72(1), 105-118. https://doi.org/ 10.1348/000709902158793

Olweus, D. (1978). Aggression in the Schools: Bullies and Whipping Boys. Oxford: England Hemisphere.

Olweus, D. (1991). Bully-victim Problems among School Children. Basic Facts and Effects of a School Based Intervention Program. En D. Pepler y K. Rubin (eds.), The Development and Treatment of Childhood Aggression (pp. 441-448). Hillsdale, N.J: Lawrence Erlbaum.

Ortega, R. (2000). Educar la convivencia para prevenir la violencia [Educating to Live Together in Order to Prevent Violence]. Madrid, España: A. Machado Libros. 
Ortega, R. y Mora-Merchán, J. A. (2000). Violencia escolar: Mito o realidad [School Violence: Myth or Reality]. Sevilla, España: Mergablum.

Piñuel, I. y Oñate, A. (2007). Acoso y violencia escolar en España [Bullying and School Violence in Spain]. Madrid, España: IIEDDI.

Polo del Río, M. I., Mendo, S., Fajardo, F. y León del Barco, B. (2017). Una intervención en aprendizaje cooperativo sobre el perfil del observador en la dinámica bullying [Intervention Effectiveness in Cooperative Learning on the Profile of the Dynamics Bullying Observer]. Universitas Psychologica, 16(1), 1-13. https://doi.org/10.11144/ Javeriana.upsy16-1.iacp

Puértolas, A. y Montiel, I. (2017). Bullying en la educación secundaria: una revisión sobre las características de las víctimas y las víctimas-acosadores y las consecuencias de su victimización [Bullying into Secondary School: A Review about Characteristics of Victims and Bully-Victims and Consequences of their Victimization]. Revista de Victimología, 5, 85-128. https://doi.org/10.12827/RVJV.5.04

Quintana, P., Montgomery, U. y Malaver, S. (2009). Modos de afrontamiento y conducta resiliente en adolescentes espectadores de violencia entre pares [Ways of Coping and Behavior in Adolescent Resilient Spectator Violence Among Peers]. Revista de Investigación en Psicología, 12(1), 35-47. https://doi.org/10.15381/rinvp.v12i1.3788

Raj, K., Aluede, O., McEachern, A. G. y Kenny, M. C. (2005). A new Perspective on Managing School Bullying: Pre-service Teacher's Attitudes. Journal of Social Sciences, 8, 43-49.

Rey, L. y Extremera, N. (2012). Inteligencia emocional percibida, felicidad y estrategias distractoras en adolescents [Perceived Emotional Intelligence, Happiness and Distracting Strategies in Adolescents]. Boletín de Psicología, 104, 87-101.
Rigby, K. (1996). Bullying in Schools - and what to Do about it. Melbourne, ACER.

Rose, C., Monda-Amaya, L. y Espelage, D. (2011). Bullying Perpetration and Victimization in Special Education: A Review of the Literature. Remedial and Special Education, 32(2), 114-130. https://doi.org/10.1177/0741932510361247

Rueda, B. y López, C. E. (2014). Música y programa de danza creativa como herramienta de expresión de emociones [Music and Creative Dancing Programme as a Tool to Transmit Emotions]. RETOS, Nuevas tendencias en Educación fisica, deporte y recreación, 24, 141-148.

Ruiz, R., Riuró, M. y Tesouro, M. (2015). Estudio del bullying en el ciclo superior de primaria [Study about Bullying in the Upper Cycle of Primary Education). Educación XXI, 18(1), 345-368. https://doi.org/10.5944/educxx1.18.1.12384

Salmivalli, C. (2010). Bullying and the Peer Group: A Review. Aggression and Violent Behavior, 15, 112-120. https://doi.org/10.1016/j.avb.2009.08.00 7

Salmivalli, C., Lagerspetz, K., Björkqvist, K., Österman, K. y Kaukiainen, A. (1996). Bullying as a Group Process: Participant Roles and their Relations to Social Status within the Group. Aggressive Behavior, 22(1), 1-15. https://doi.org/10.1002/ (SICI) 1098-2337(1996)22:1\%3C1::AID$\mathrm{AB} 1 \% 3 \mathrm{E} 3.0 . \mathrm{CO} ; 2-\mathrm{T}$

Salovey, P., Mayer, J. D., Goldman, S. L., Turvey, C. y Palfai, T. P. (1995). Emotional Attention, Clarity, and Repair: Exploring Emotional Intelligence using the Trait Meta-Mood Sacale. En J. K. Pennebaker (eds.), Emotion, Disclosure, and Health (pp. 125154). Washington: American Psychological Association.

Salovey, P. y Mayer, J. D. (1990). Emotional Intelligence. Imagination, Cognition, and Personality, 
9(3), 185-211. https://doi.org/10.2190/DUGGP24E-52WK-6CDG

Santos, A. y Romera, E. M. (2013). Influencia de la exposición a la violencia en conductas de agresión en cyberbullying [Influence of Exposure to Violence on Behavior of Aggression in Cyberbullying]. Apuntes de Psicología, 31(2), 225-235.

Sierra, C. (2009). Manifestaciones de violencia en la escuela primaria: elementos de perfilación de víctimas y agresores [Manifestations of Violence in Primary School: Elements of Profiling Victims and Aggressors]. Revista Panorama, 7, 98-116.

Stephenson, P. y Smith, D. (1987). Anatomy of a Playground Bully. Education, 18, 236-237.

Storch, E. A., Heidgerken, A. D., Adkins, J. W., Cole, M., Murphy, T. K. y Geffken, G. R. (2005). Peer Victimization and the Development of Obsessive-
Compulsive Disorder in Adolescence. Depression and Anxiety, 21(1), 41-44. https://doi.org/10.1002/ da. 20040

Sullivan, K., Cleary, M. y Sullivan, G. (2005). Bullying en la enseñanza secundaria. El acoso escolar cómo se presenta y cómo afrontarlo [Bullying in Secondary Education. The School Harassment how it Occurs and how to Deal with it]. Barcelona, España: CEAC.

Trautmann, A. (2008). Maltrato entre pares o "bullying". Una visión actual [Abuse among Partners or "Bullying". Current Concepts]. Revista Chilena de Pediatría, 79(1), 13-20. https://doi.org/10. 4067/S0370-41062008000100002

\title{
EMOTIONAL REPERCUSSIONS OF SCHOOL VIOLENCE: INFLUENCE ON EMOTIONAL INTELLIGENCE
}

\author{
NIEVES GUTIÉRREZ ÁNGEL ${ }^{1}$
}

\section{EXTENDED SUMMARY}

Bullying is not a fixed or stable dynamic, but it can be described as a five-phase process that makes reference to the process in which a victim faces an abuse situation at school (Piñuel y Oñate, 2007). This process begins at the incident, through the own abuse and stigmatization. The origin and the pain latency damages the victim and as a consequence of it, the victim experiences somatic or psychological manifestations leading to inferiority complex or exclusion. Olweus (1978) defines both bullying and its main characters for the first time (Ruiz et al., 2015): the abuser, the victim and the observer.
Following Sullivan, Cleary, and Sullivan (2005), five levels were also made in the propagation process that leads to aggression. In this sense, it shares with the previous process the first level or step, which is about the incidence of one's own harassment. Next, all the personnel that surrounds the victim intervene in it, both in the school context (classmates, teachers ...) and in the family context. Therefore, as of this moment, it ceases to be an act between the aggressor and the victim only. The following levels are related to taking into account the facts, and the proposal of the different types of attention to the occurrence of them.

It is therefore Olweus (1978), who defines and identifies both bullying and its protagonists for the first time 
(Ruiz et al., 2015), that is, aggressor, victim and observer. Attending to each of them, the aggressor is the one who performs the harmful action, the victim who is subjected to such action and the injured, and the observer, who is a mere curious accomplice of the situation.

Bearing in mind all these roles, the abusers are considered impulsive students, with barely self-control (Martos y del Rey, 2013), low frustration tolerance and difficulties complying the rules (Díaz-Aguado, 2006). They enjoy attacking other people for no reason at all. Also, they tend to associate attacking with popularity and believe that there are people that may deserve it.

As for the physical characteristics, they normally are older and physically stronger than their victims (Ruiz et al., 2015). They have a negative attitude towards school (Martos y del Rey, 2013), as well as low school performance and negative relationships with the faculty.

Regarding the victims, they usually are isolated students with low level of acceptance and high level of rejection (Sierra, 2009), they are anxious, vulnerable and insecure. They do not have the ability to react and they have low self-esteem most of the time. This lack of security and assertiveness leads to a negative vision of themselves (Albadalejo, 2011), a poor type of personality or some different physical traits (Armero et al., 2011; Díaz-Aguado et al., 2013).

Concerning the academic field, the fact of being involved as a victim may affect or not the academic performance. The victim may feel fear or neglect at school (Martos y del Rey, 2013) or even suffer psychosomatic disorders such as pain, insomnia or retraction (Rigby, 1996). They usually share the powerful silence in the face of what is happening.

In addition to the own characteristics of each role, the existing literature also shows the consequences that each role may face (Garaigordobil, 2017; Lara-Ros et al., 2017; Larrañaga et al., 2013; Morales et al., 2016). These consequences correlate with the tendency that using violence leads to delinquency in the abuser's case (Ortega, 2000). The victims suffer exclusion and struggle to interact with people (Martínez-Otero, 2005). In addition they have de- valuation feelings, anxiety, dissatisfaction, guilty, loneliness, phobia or insecurity that cause them negative feelings (Morris et al., 2006; Storch et al., 2005). Negative behaviours are associated with hostility and aggression (Garaigordobil, 2015; Santos y Romera, 2013) and low levels of empathy presence may give room to psychosocial and psychopathological issues (Garaigordobil, 2011) because of the abuser's thought that school violence is a usual thing (Puértolas y Montiel, 2017).

In any case, the consequences of these acts affect the three roles involved underlining the high probability of suffering psychosocial or psychopathological problems (Ruiz et al., 2015). Although the worst consequences of this phenomenon would be suicide, the rest of the consequences are not less important because they could affect the health, the quality of life, the well-being and the personal development of the ones involved (Garaigordobil, 2013).

The aim of this work is to identify the influence of being involved in bullying inside the levels of Emotional Intelligence of each role.

The method used is correlated with an ex post factum design. Its nature is retrospective and comparative given the fact that the three roles are being compared inside the bullying dynamics (abuser, victim and observer) with a dependent variable that corresponds to every level of Emotional Intelligence.

The sample consists of third and fourth grade students of Social Education Degree from the University of Almería (Spain), which have been selected through a simple random sampling with 175 subjects.

The sample average age was 25.79 years with a standard deviation $\mathrm{SD}=8.01$. Regarding the sex the $22.9 \%$ $(\mathrm{N}=40)$ were men and the $77.1 \%(\mathrm{~N}=135)$ were women. $49.2 \%(\mathrm{~N}=75)$ were in third grade and $57.1 \%(\mathrm{~N}=100)$ in fourth grade.

Moreover, three instruments were used. The first one would be an instrument developed ad hoc, which is to collect sociodemographic information about the sample re- 
garding age, sex, degree or training inside of the educational context.

The second one used is the Trait Meta Mood Scale-24 (TMMS-24) developed by Fernández-Berrocal et al. (2004), which corresponds to an adaptation of the Trait Meta-Mood Scale (TMMS) developed by Salovey et al. (1995). Its purpose is to evaluate Emotional Intelligence perceived by the subject.

The third one used is the survey about abuse between equals at school (Nicolaaides et al., 2002). Its main aim is to evaluate the different aspects of school violence taking as a reference the professionals education's view.

For this work in particular, above all the items they make it itself, there were only used the ones assigned to the collection of information related to the own experience as an abuser, victim and observer.

The data analysis was carried out through descriptive statistical analysis regarding each role. In addition to Student $t$ tests for independent groups, in this case applied with variables such as each of the aforementioned roles. The purpose of all of these tests was not only to measure the scores obtained in terms of the perceived Emotional Intelligence, but also to analyze the possible existence of differences between each group. Finally, in those situations in which statistically significant differences were found, the Cohen d (1988) was calculated to measure the effect size. All this through the use of the statistical analysis program SPSS in its version 23.

As for the results obtained from this work, they exhibit the fact that there are no differences between Emotional Intelligence and being or not the victim. Nevertheless, we must be cautious about these results considering that inside of all the dimensions of Emotional Intelligence, the ones that obtain bigger levels of attention, clarity and emotional regulation are the no victims.

Furthermore, regarding the abuser's role, the no abusers are the ones who show bigger levels of attention, clarity and emotional regulation existing differences statistically significant in the case of emotional attention. Considering the results, the abusers are the ones with a low capacity for self-criticism, rare social adjustment, big need for self-affirmation... (Albadalejo, 2011).

Lastly, as for the observer role, they obtain bigger levels of emotional reparation and clarity (this one is significant) than the observers. Only occurs the opposite when it comes to emotional attention.

In conclusion, the results of this work show that both victims and abusers have higher levels of each Emotional Intelligence dimension. If we address this hypothesis carefully, it leads us to think that being involved in any school violence incident -whether as a victim or abuser- has a negative influence on the levels of Emotional Intelligence because it reduces them.

Without putting aside the observers, who are the ones that depending on their position to the facts, they show higher or lowers levels of such levels.

For all these reasons, the results provided by this work highlight the fact that those people who do not intervene in episodes related to school violence, in this case both nonvictims and non-aggressors, have higher levels in each case. one of the dimensions that make up the Emotional Intelligence (attention, clarity and emotional regulation). Concluding, therefore, that the fact of being a protagonist in an episode related to school violence, either as a victim or as an aggressor, has a negative influence on Emotional Intelligence levels, since it decreases them. However, in the case of the role of observers, the increase or decrease in levels of Emotional Intelligence depend on the positioning they adopt in the face of these events. For there will be higher or lower levels of Emotional Intelligence, depending on whether they adopt a more active or passive role within this same role. 